\title{
A synergistic behavior underpins human hand grasping force control during environmental constraint exploitation
}

\author{
Giuseppe Averta ${ }^{1,2,3}$, Edoardo Battaglia ${ }^{1,3}$, Cosimo Della Santina ${ }^{1,3}$, Manuel G. Catalano ${ }^{2}$ and Matteo Bianchi ${ }^{1,3}$
}

\begin{abstract}
Despite the complex nature of human hands, neuroscientific studies suggested a simplified kinematic control underpinning motion generation, resulting in principal joint angle co-variation patterns, usually called postural hand synergies. Such a low dimensional description was observed in common grasping tasks, and was proven to be preserved also for grasps performed by exploiting the external environment (e.g., picking up a key by sliding it on a table). In this paper, we extend this analysis to the force domain. To do so, we performed experiments with six subjects, who were asked to grasp objects from a flat surface while force/torque measures were acquired at fingertip level through wearable sensors. The set of objects was chosen so that participants were forced to interact with the table to achieve a successful grasp. Principal component analysis was applied to force measurements to investigate the existence of co-variation schemes, i.e. a synergistic behavior. Results show that one principal component explains most of the hand force distribution. Applications to clinical assessment and robotic sensing are finally discussed.
\end{abstract}

\section{INTRODUCTION}

The human hand is a very complex biomechanical system, with high capabilities in terms of dexterity and force control. However, the Central Nervous System (CNS) seems to deal with such a complexity by leveraging on dimensionality reduction strategies. Indeed, several neuroscientific studies reported on an underlying low-dimensionality control, typically referred to as synergies, which can be observed at different levels, e.g. kinematic [1], muscular [2], and neural [3]. From a kinematic point of view, while a lot of effort has been devoted to describing the synergistic control that modulates hand movements during grasp [1], the relationship between postural hand synergies and contact force distribution and how the interaction with the external environment shapes synergistic control is still debated [4], [5], [6]. In a previous work we studied how kinematic synergistic control is modified when the hand interacts with the environment to grasp an object [5], and proposed a consequent unsupervised clustering of principal hand approaches [7]. in this paper we further extend this analysis to the force domain. Our analysis is based on force and torque $(\mathrm{F} / \mathrm{T})$ measurements at fingertip

This project has received funding from the European Union's Horizon 2020 research and innovation programme under grant agreement No. 688857 (SoftPro) and 645599 (Soma). The content of this publication is the sole responsibility of the authors. The European Commission or its services cannot be held responsible for any use that may be made of the information it contains.

1 Centro di Ricerca "Enrico Piaggio", Università di Pisa, Largo Lucio Lazzarino 1, 56126 Pisa, Italy (corresponding author to provide e-mail: giuseppe.averta@ing.unipi.it)

2 Soft Robotics for Human Cooperation and Rehabilitation, Fondazione Istituto Italiano di Tecnologia, via Morego, 30, 16163 Genova, Italy

${ }^{3}$ Dipartimento di Ingegneria dell'Informazione, Università di Pisa, Largo Lucio Lazzarino 1, 56126 Pisa, Italy
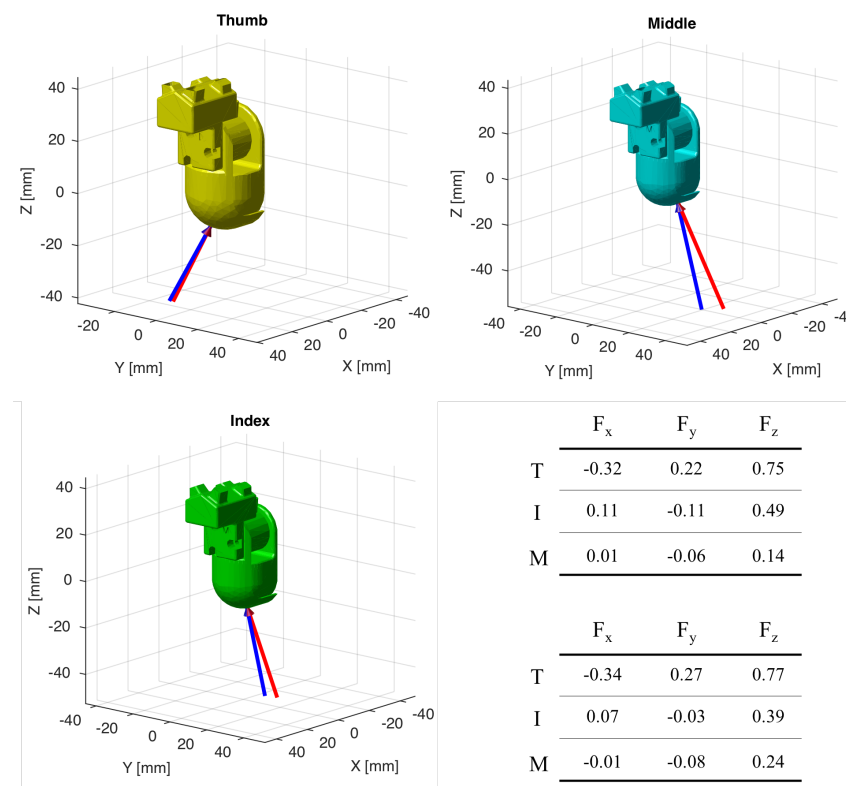

Fig. 1. First force synergy for the three fingers. In blue the contact phase, in red the flight phase. On the right-bottom, in top table we report the numerical values of the first synergy in the contact phase while in the bottom table we report the values for the flight phase. Each row refers to one finger, each column refers to the direction. Please note that all coefficients are dimensionless.

level for thumb, index and middle fingers during grasping of a set of objects from a flat surface, and uses Principal Component Analysis (PCA) to identify the principal force directions or synergies. This investigation is motivated by the need of developing new tools for the evaluation of physiological and pathological behavior in object grasping and manipulation. More specifically, some pathologies such as stroke can alter subject grasping capabilities in everyday-life, eventually resulting in different synergistic behavior in force distribution. At the same time, the study reported in this work could inspire simplified sensing guidelines for robotic hands and prostheses. The latter point is particularly important when considering soft robotic devices, which deform with the environment as humans actually do.

\section{Materials And Methods}

Experiments - We asked six able-bodied, right handed volunteers (three females; age $25.17 \pm 2$ years) to reach and grasp objects, which were a set of 21 items suitably selected to force the interaction with the environment (a complete list, here omitted for the sake of space, can be found in [5]). Each finger was endowed with a ThimbleSense [8], which 


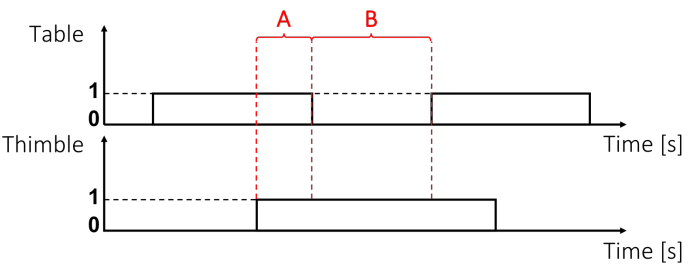

Fig. 2. In the top graph we show an ideal plot of signal presence on table sensor ( 1 if over a treshold, 0 otherwise), while in the bottom graph we report the analogous for ThimbleSense recordings. We identify two main regions of interest for our analysis: (A) in which the hand is in contact with the table; (B) in which the hand has lifted the object.

consists of two rigid shells assembled around a force and torque sensor, allowing to retrieve contact point information through Intrinsic Tactile Sensing. For each trial, participants were asked to reach and grasp the object placed at the center of a flat surface, lift and hold it for one second, then put it back on the table. Two trials were performed for each of the 21 objects.

Pre-Processing - We extrapolated two ranges of interest from the whole task execution: (A) in which the hand is in contact with the table and (B) in which the hand has lifted the object (see Fig. 2). Regarding phase (A), during which the interaction with the object has the goal of obtaining a stable grasp, we analyze the last five frames, which contain information on the force exchange immediately before lift. These F/T vectors are then averaged to get a single vector for each acquisition. Regarding phase (B), we extract a single $\mathrm{F} / \mathrm{T}$ vector as mean between all the frames. With this procedure we extrapolate, for each task, for each subject and for each experimental phase $(\mathrm{A}, \mathrm{B})$, one representative $\mathrm{F} / \mathrm{T}$ vector $\in \mathbf{R}^{m}$, containing $3 \mathrm{D}$ forces at fingertip level. Note that, although we recorded forces and torques during the experiments, for the analysis we used only the force components (i.e. $m=9$ ), since the torques appear to be negligible w.r.t. the forces.

Data Analysis - For each experimental phase (A,B), we collected the $\mathrm{F} / \mathrm{T}$ vectors in a matrix $X_{A, B} \in \mathbf{R}^{n, m}$, where $n=252$ is the number of trials. Principal Components (PCs) are calculated as eigenvectors of the covariance matrix $S=X_{A, B} X_{A, B}^{T}-\mu \mu^{T}$, where $\mu=\operatorname{mean}\left(X_{A, B}\right)$. The variance explained by each PC is calculated as the normalized corresponding eigenvalue.

\section{RESUlts AND Discussions}

Principal Component Analysis (PCA) on the two experimental phases shows that one predominant Principal Component exists and explains over the $60 \%$ of the total dataset variability (see Fig. 3). In Fig. 1 we plot the first $\mathrm{PC}$ as direction of forces insisting on thumb, index and middle fingers (in blue the contact phase (A), in red the flight phase (B)). As expected the first PC appears with a strong dominance of direction along the axis normal to the fingertip surface. What is noticeable is that the coordination between fingers appears maintained in both the experimental phases, with slight variations mainly on index and middle finger probably due to the effect of the object weight.

These results suggest that a coordination between finger forces exists in grasping tasks, and that this behavior is

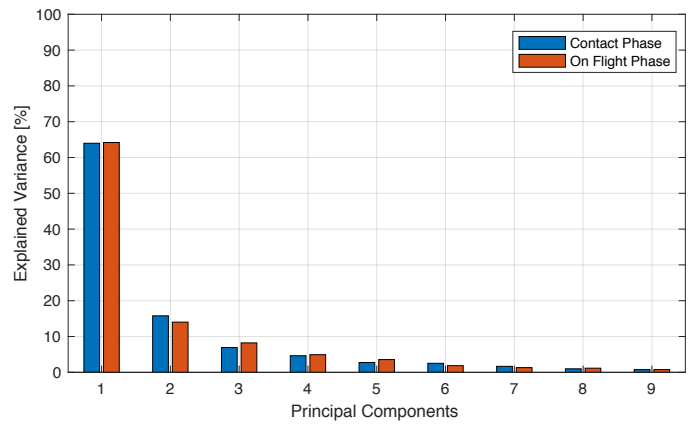

Fig. 3. Variance explained by each PC. In blue the contact phase, in red the flight phase. A predominant first synergy explaining more than the $60 \%$ of the data can be observed in both phases.

preserved also in case of interaction with the environment. This hypothesis is further confirmed by the fact that the first PC plays a crucial role in explaining the force distribution, with an explained variance higher than $60 \%$ in both the experimental cases. We believe that these finding could be used to develop new assessment procedures, e.g. in poststroke patients, where the pathology could alter the synergistic behavior thus resulting in different principal components. At the same time, the co-variation in force distribution could be employed to suggest optimal sensing strategies for soft robotic hands, that deform as human hands actually do , when constraints on the number and quality of sensors are present, similarly to what was done in [9] for kinematic under-sensing techniques of hand poses relying on postural synergies.

\section{REFERENCES}

[1] M. Santello, M. Flanders, and J. F. Soechting, "Patterns of hand motion during grasping and the influence of sensory guidance," Journal of Neuroscience, vol. 22, no. 4, pp. 1426-1435, 2002.

[2] A. d'Avella, P. Saltiel, and E. Bizzi, "Combinations of muscle synergies in the construction of a natural motor behavior," Nature neuroscience, vol. 6, no. 3, p. 300, 2003.

[3] M. C. Tresch, P. Saltiel, and E. Bizzi, "The construction of movement by the spinal cord," Nature neuroscience, vol. 2, no. 2, pp. 162-167, 1999.

[4] G. Averta, F. Angelini, M. Bonilla, M. Bianchi, and A. Bicchi, "Incrementality and hierarchies in the enrollment of multiple synergies for grasp planning," IEEE Robotics and Automation Letters, 2018.

[5] C. Della Santina, M. Bianchi, G. Averta, S. Ciotti, V. Arapi, S. Fani, E. Battaglia, M. G. Catalano, M. Santello, and A. Bicchi, "Postural hand synergies during environmental constraint exploitation," Frontiers in neurorobotics, vol. 11, p. 41, 2017.

[6] M. Santello, G. Baud-Bovy, and H. Jörntell, "Neural bases of hand synergies," Frontiers in computational neuroscience, vol. 7, p. 23, 2013.

[7] G. Averta, C. Della Santina, E. Battaglia, S. Ciotti, V. Arapi, S. Fani, and M. Bianchi, "From humans to robots: The role of cutaneous impairment in human environmental constraint exploitation to inform the design of robotic hands," in Ultra Modern Telecommunications and Control Systems and Workshops (ICUMT), 2017 9th International Congress on. IEEE, 2017, pp. 179-184.

[8] E. Battaglia, M. Bianchi, A. Altobelli, G. Grioli, M. G. Catalano, A. Serio, M. Santello, and A. Bicchi, "Thimblesense: a fingertip-wearable tactile sensor for grasp analysis," IEEE transactions on haptics, vol. 9, no. 1, pp. 121-133, 2016.

[9] M. Bianchi, P. Salaris, and A. Bicchi, "Synergy-based hand pose sensing: Optimal glove design," The International Journal of Robotics Research, vol. 32, no. 4, pp. 407-424, 2013. 